\title{
Activity of anti-CR3-RP polyclonal antibody against biofilms formed by Candida auris, a multidrug-resistant emerging fungal pathogen
}

\author{
Jaroslava Dekkerová ${ }^{1}$. Jose L. Lopez-Ribot ${ }^{2} \cdot$ Helena Bujdáková ${ }^{1}$
}

Received: 12 July 2018 / Accepted: 3 October 2018 / Published online: 16 October 2018

(C) Springer-Verlag GmbH Germany, part of Springer Nature 2018

\begin{abstract}
Fungal biofilm has remained a serious medical problem that complicates treatment of mycoses. In particular, once biofilms are formed, they display high levels of resistance against most common antifungals. Candida auris is currently considered as a serious emerging fungal pathogen frequently exhibiting high levels of resistance to antifungals. Recent studies have confirmed that $C$. auris shares similarity with Candida albicans in regards to virulence-associated proteins involved in adherence and biofilm development. Complement receptor 3-related protein (CR3-RP) is one of the key surface antigens expressed by Candida species during biofilm formation. Here, we have investigated the presence of this cell surface moiety on the surface of $C$. auris, as well as the potential of anti-CR3-RP polyclonal antibody $(\mathrm{Ab})$ to inhibit biofilm formation by this emerging fungal pathogen. Using indirect immunofluorescence and ELISA, we were able to confirm the presence of CR3-RP in C. auris cells within biofilms. Further, not only anti-CR3-RP Ab was able to inhibit biofilm formation by multiple $C$. auris strains when added during the adherence phase, but it also demonstrated activity against $C$. auris 24-h pre-formed biofilms, which compared favorably to levels of inhibition achieved by treatment with current conventional antifungals fluconazole, amphotericin B, and caspofungin. Overall, our data demonstrate the presence of this antigen on the surface of C. auris and points to the potential of anti-CR3-RP Ab in eradication of biofilms formed by this novel fungal pathogen.
\end{abstract}

Keywords CR3-RP $\cdot$ Polyclonal antibody $\cdot$ Biofilm $\cdot$ Resistance $\cdot$ Candida auris

\section{Introduction}

Complement receptor 3-related protein (CR3-RP) is an immunogenic surface protein expressed on the cell wall of Candida species (spp.) during adherence and biofilm formation, with functional and structural similarity to the human complement

Electronic supplementary material The online version of this article (https://doi.org/10.1007/s10096-018-3400-x) contains supplementary material, which is available to authorized users.

Helena Bujdáková

helena.bujdakova@uniba.sk

1 Department of Microbiology and Virology, Faculty of Natural Sciences, Comenius University in Bratislava, Ilkovičova 6, 84215 Bratislava, Slovakia

2 Department of Biology and South Texas Center for Emerging Infectious Diseases, The University of Texas at San Antonio, San Antonio, TX 78249, USA receptor 3 (CR3) expressed on neutrophils, macrophages, and monocytes [1-4]. Recently, our group has demonstrated an important role of CR3-RP in adherence and biofilm development of different Candida spp. Anti-adhesive and anti-biofilm effects of anti-CR3-RP polyclonal antibody (Ab) directed against the antigen CR3-RP on Candida cell wall have already been described [5-7]. Most recently, a reduction in adherence and biofilm formation was proven not only in Candida albicans, but also in Candida dubliniensis after treatment of the yeast cells with anti-CR3-RP Ab in vitro/ex vivo and in vivo [8].

Candida auris is considered an emerging fungal pathogen, spreading rapidly around the world, and increasingly associated with nosocomial invasive fungal infections which very often are resistant to treatment with current conventional antifungals [9-12]. It has already been proven that this species (sp.) is able to colonize substrates and subsequently form biofilms in vitro and in vivo [12-14]. The genome of C. auris contains genes encoding for two types of efflux pumps, ABC transporters and major facilitators $[15,16]$, 
which could explain why $C$. auris often displays multidrug resistance to various antifungals $[17,18]$. Moreover, $C$. auris expresses many genes encoding virulence-associated proteins similar to those expressed in C. albicans, some of which have been associated with invasion and biofilm development [17]. CR3-RP is believed to be one of the important surface antigens involved in virulence-associated processes. Beside C. albicans and C. dubliniensis, this protein was also identified in C. parapsilosis, C. glabrata, and C. krusei $[2,8]$. Antibodies directed against surface antigens are considered to be potential candidates for the development of novel approaches for the therapy of Candida infections [19-21]. In the case of anti-CR3-RP Ab, its potential anti-adherence and antibiofilm effect have already been described in C. albicans and C. dubliniensis by our group [7, 8]. Mentioned results were a motivation for continuation in this research using C. auris. Thus, the main aims of this research were to investigate the presence of CR3-RP on the surface of $C$. auris and to evaluate the potential of anti-CR3-RP Ab in eradication of multidrugresistant $C$. auris biofilms.

\section{Material and methods}

\section{Candida strains and growth conditions}

C. albicans wild-type strain SC5314 [22] was used as control in all experiments. The C. auris clinical isolates 0390, 0383, and 0386 with different patterns of resistance to antifungals were obtained from the Centers for Diseases Control and Prevention (CDC) Antimicrobial resistance bank (USA). Prior to all experiments, cells from stocks stored at $-80{ }^{\circ} \mathrm{C}$ were streaked onto a yeast extract-peptone-dextrose plate (YPD) supplemented with 2\% of agar (Becton Dickinson, USA) and incubated overnight at $30{ }^{\circ} \mathrm{C}$. One loopful of cells from YPD agar plates was inoculated into flasks containing $20 \mathrm{ml}$ of YPD broth and grown in an orbital shaker (180 rpm, New Brunswick Scientific, USA), for up to $16 \mathrm{~h}$ at $30^{\circ} \mathrm{C}$. The cells were then washed twice with phosphate-buffered saline (PBS) buffer (Sigma, USA) and adjusted to the appropriate density for each experiment.

\section{Antifungal susceptibility of biofilms formed by $C$. albicans and C. auris strains}

All strains were tested for their ability to form biofilm (Supplementary Material Fig. S1). The susceptibility of biofilms against fluconazole (FLU, SAGENT Pharmaceuticals, USA), caspofungin (CAS, Merck, USA), and amphotericin B (AMB, Sigma, USA) was tested in vitro following the 96-well microtiter plate method first described by Ramage et al. [23] with slight modifications. The initial inoculum was adjusted to $2 \times 10^{6}$ cells per $\mathrm{ml}$ in RPMI 1640 medium containing $2 \%$ D-glucose (Corning, USA) and buffered with $165 \mathrm{mM}$ morpholine propanesulfonic acid (MOPS; Sigma, USA) to $\mathrm{pH}$ 7.0. The anti-biofilm activity of the antifungals was tested in two different modalities: (1) agents were added at the beginning of biofilm formation and (2) antifungal drugs were added to 24 -h pre-mature biofilms. In both treatment modalities, the plates were washed after incubation, and the viability of cells within the biofilms was evaluated using an XTT (2, 3-bis (2-methoxy-4-nitro-5-sulfophenyl)2Htetrazolium-5-carboxanilide sodium salt, Sigma, USA) reduction assay as previously described [23]. Results were calculated as a mean value \pm standard deviation (SD) from at least five parallel wells and from two independent experiments. Each experiment contained positive control (biofilm without drug, to allow for uninterrupted biofilm formation) and negative control (no cells, to monitor contamination and to be able to calculate the percent inhibition). The extent of biofilm inhibition was calculated as a percentage of colorimetric readings of biofilm cells in treated wells and compared to the control sample without agents, which was set to $100 \%$. From these values, the "sessile minimum inhibitory concentrations" (SMICs) was calculated at 50\% (for FLU and CAS) and $100 \%$ inhibition (for AMB) as per Ramage et al. [23].

\section{Isolation and quantification of CR3-RP antigen in $C$. auris protein lysates}

The presence of CR3-RP was determined in biofilm cells of C. auris using indirect immunofluorescence, and levels of expression of CR3-RP were quantified using an ELISA assay (enzyme-linked immunosorbent assay), according to the protocols previously published by our group $[8,24]$ with some modifications. For this work, one representative $C$. auris strain (C. auris 0390) was selected based on biofilm production and resistance profile. The strain C. albicans SC5314 was used as the control. Fluorescence was detected by inverted fluorescence microscope ZEISS Axio Observer 5 (Zeiss, Germany) (excitation/emission spectra 485/515 nm). The pictures were captured by ZEISS Axiocam 503 (Zeiss, Germany) and evaluated by software ZEN Pro (Zeiss, Germany). The level of CR3-RP was quantified in protein lysates of the tested strains using an ELISA assay as previously described by Chupáčová et al. [8]. Proteins were extracted from (i) planktonic cells from overnight yeast suspension, (ii) biofilm cells after 24-h cultivation, and (iii) biofilm cells after 48-h cultivation. Results were calculated as a mean value \pm SD from at least five parallel wells and two independent experiments.

\section{Anti-biofilm activity of anti-CR3-RP Ab}

The inhibitory effect of anti-CR3-RP Ab in biofilm formation as well as its activity against preformed biofilms was tested following mostly the same protocol described above for 
antifungal susceptibility testing [23], but the diluted anti-CR3$\mathrm{RP} A \mathrm{~b}$ was tested instead of antifungal agents. For inhibition of biofilm formation, $100 \mu \mathrm{l}$ of anti-CR3-RP Ab (dilution 1:50 in fresh RPMI-MOPS) was added to the wells, and plates were incubated for $90 \mathrm{~min}$ at $37^{\circ} \mathrm{C}$. After the adherence phase, nonadhered cells were removed and adherent cells were washed twice with PBS. Adherent cells were then overlaid again with $100 \mu \mathrm{l}$ of anti-CR3-RP Ab and incubated at $37^{\circ} \mathrm{C}$ for a further $48 \mathrm{~h}$. For activity against 24 -h pre-formed biofilms, $100 \mu \mathrm{l}$ of the diluted anti-CR3-RP Ab was added to wells. Plates were incubated for another $24 \mathrm{~h}$ at $37^{\circ} \mathrm{C}$. The XTT reduction assay was performed as described above. Each experiment was performed in five parallel wells and performed twice. Data were expressed as mean values $\pm \mathrm{SD}$.

\section{Microscopy}

For microscopy, biofilms of $C$. auris 0390 and C. albicans SC5314 were prepared using a similar protocol as above, but instead using 24-well microtiter plates (Corning Inc., USA) with a final volume of $500 \mu \mathrm{l}$ in each well. The final dilution of anti-CR3-RP Ab was 1:100 in RPMI-MOPS and $\mathrm{Ab}$ was added to the cells at $t=0 \mathrm{~h}$ (for inhibition of biofilm formation) and $t=24 \mathrm{~h}$ (for activity against pre-formed biofilms). RPMI-MOPS medium without $\mathrm{Ab}$ was used as a control. After $48 \mathrm{~h}$, the medium was aspirated, the biofilms were gently washed with PBS and stained with crystal violet solution (0.6 g crystal violet, Sigma, USA; prepared in $10 \mathrm{ml}$ of isopropanol, $10 \mathrm{ml}$ of methanol, and $180 \mathrm{ml}$ of Millipore water). After $5 \mathrm{~min}$, the crystal violet was removed and plates were washed twice with $500 \mu$ of distilled water. Samples were directly observed using a $40 \times$ objective in an inverted system microscope (Westover Scientific, USA) equipped for photography. The images were processed for display using Micron software (Westover Scientific, USA).

\section{Statistical analyses}

Results were evaluated by statistical analysis using one-way $t$ test using Graph Pad Prism software (Graph Pad, San Diego, USA). Differences were considered statistically significant at $p<0.05(*)$, highly significant at $p<0.01(* *)$, and extremely significant at $p<0.001(* * *)$.

\section{Results and discussion}

The anti-biofilm effect of anti-CR3-RP Ab has been described recently in some Candida spp. [7, 8] and this work represents a continuation of those experiments, with a focus on the effectiveness of $\mathrm{Ab}$ against the multidrug-resistant pathogen C. auris, in particular in biofilm formed in vitro.

\section{Resistance of $C$. auris biofilms against commercial antifungal agents}

Formation of fungal biofilms represents a major medical problem mostly due to their recalcitrance to antifungal treatment [25] as cells within the biofilms are up to 1000-fold more resistant compared to their non-biofilm, planktonic counterparts [23, 26, 27]. Our initial experiments demonstrated that all $C$. auris isolates tested were able to form biofilm in vitro, but to a lower extent than $C$. albicans (Supplementary Fig. $\mathrm{S} 1$ ). The results are similar to those reported by Sherry et al. [13] for a different $C$. auris strains. In agreement with mentioned study, our results confirmed the decreased susceptibility of $C$. auris biofilms to current antifungals FLU, CAS, and AMB (Table 1). When drugs were added at the beginning of biofilm formation during the adherence phase $(t=0 \mathrm{~h})$, FLU was the least effective drug against all $C$. auris isolates $\left(\mathrm{SMIC}_{50}>64 \mu \mathrm{g} / \mathrm{ml}\right)$. $\mathrm{SMIC}_{50}$ values for CAS were determined to be $0.5-1 \mu \mathrm{g} / \mathrm{ml}$. Interestingly, C. auris 0390 also demonstrated a higher levels of resistance against AMB. When the antifungal drugs were added to the 24 -h pre-formed biofilm, results clearly indicated the intrinsic resistance of biofilms formed by all three tested $C$. auris isolates (Table 1). These results confirmed that eradication of C. auris biofilms by conventional drugs is problematic and searching for alternative approaches seems to be essential.

\section{C. auris expresses CR3-RP antigen}

The expression of CR3-RP has already been observed in some Candida spp. [2, 8, 28]. Therefore at first, it was important to demonstrate whether CR3-RP is expressed by $C$. auris as well. As it is shown in Fig. 1, indirect immunofluorescence using the anti CR3-RP Ab demonstrated the expression of this antigen on the surface of $C$. auris cells, with fluorescence levels comparable to those observed for the $C$. albicans

Table 1 Susceptibility profiles of $C$. auris biofilms to conventional antifungals applied during the adherence phase for inhibition of biofilm formation $(t=0 \mathrm{~h})$ or to 24-h pre-formed biofilm for activity against preformed biofilms $(t=24 \mathrm{~h})$. Values are in $\mu \mathrm{g} / \mathrm{ml}$

\begin{tabular}{|c|c|c|c|c|c|c|}
\hline \multirow[t]{2}{*}{ Strains } & \multicolumn{3}{|c|}{$t=0 \mathrm{~h}$} & \multicolumn{3}{|c|}{$t=24 \mathrm{~h}$} \\
\hline & FLU & CAS & AMB & FLU & CAS & $\mathrm{AMB}$ \\
\hline C. auris 0390 & $>64$ & 1 & $>8$ & $>64$ & $>16$ & $>8$ \\
\hline C. auris 0383 & $>64$ & 1 & 1 & $>64$ & $>16$ & $>8$ \\
\hline C. auris 0386 & $>64$ & 0.5 & 1 & $>64$ & $>16$ & $>8$ \\
\hline
\end{tabular}

Values for FLU and CAS are expressed as $\mathrm{SMIC}_{50}$, minimal inhibitory concentrations inhibiting metabolic activity of sessile cells by $50 \%$ or more compared to the control sample without agent

Values for $\mathrm{AMB}$ are expressed as $\mathrm{SMIC}_{100}$, minimal inhibitory concentration of $\mathrm{AMB}$ inhibiting metabolic activity of sessile cells by $100 \%$ compared to the control sample without agent 

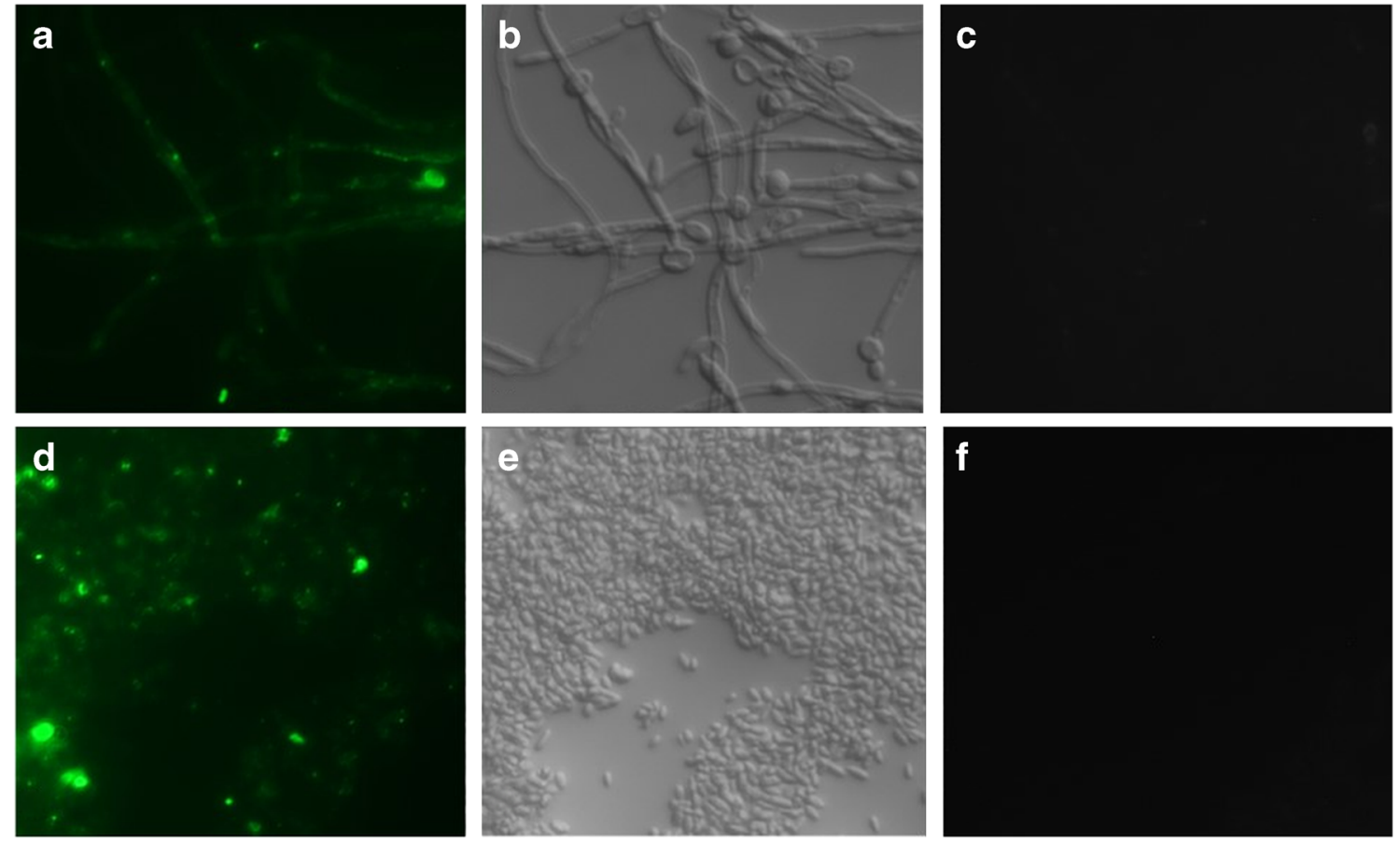

Expression of CR3-RP

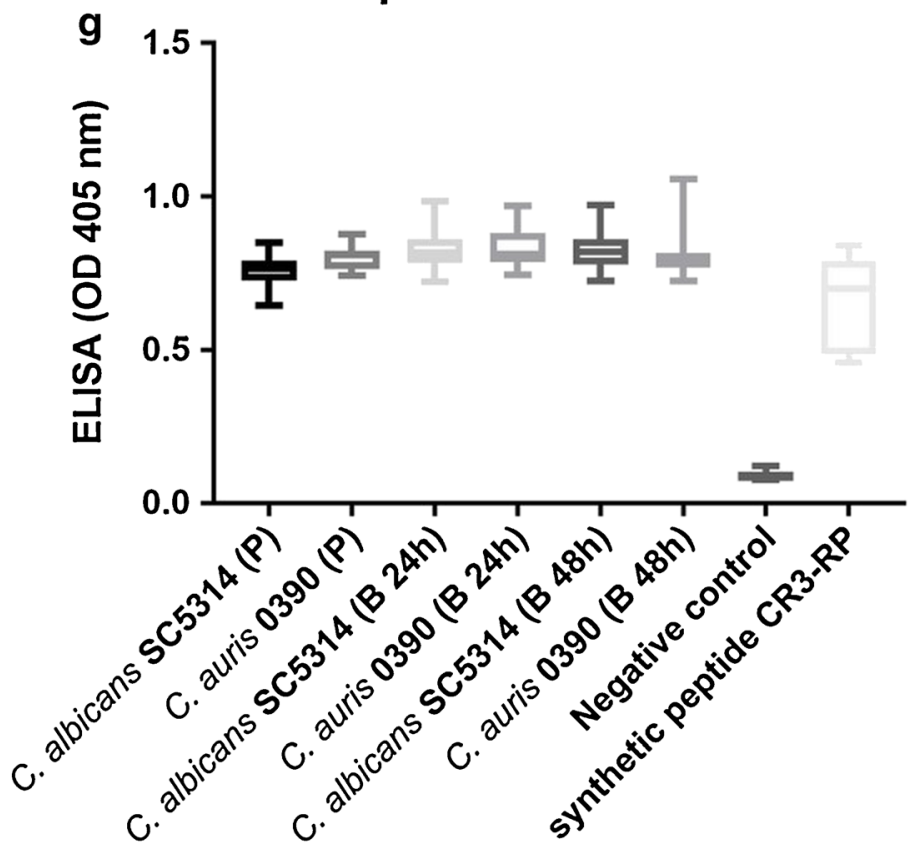

Fig. 1 Expression of the CR3-RP antigen in cells from biofilms of C. albicans SC5314 (a) and C. auris 0390 (d) as visualized by indirect immunofluorescence using the anti-CR3-RP Ab. b, e Bright filed images; c, f negative controls without primary antibody. Magnification $400 \times . \mathbf{g}$

control cells (Fig. 1a, d). The same anti-CR3-RP Ab was used in an ELISA assay in order to quantity levels of CR3-RP. As it is also seen in Fig. 1g, results of this ELISA corroborated the presence of CR3-RP in C. auris lysate to similar level compared to $C$. albicans. Additionally, these results proved slightly increased quantity of CR3-RP in 24-h pre-matured biofilm
Results of ELISA to quantity levels of CR3-RP in protein lysates of planktonic and biofilm cells of C. albicans SC5314 and C. auris 0390. Negative control — samples without Ab; positive control—synthetic peptide CR3-RP in concentration of $1 \mu \mathrm{g} / \mathrm{ml}$

compared to the planktonic cells for both $C$. albicans and C. auris; however, differences were not statistically significant. The image from indirect immunofluorescence also confirmed the presence of CR3-RP in biofilms of both spp. It was initially believed that the expression of CR3-RP was mainly associated with mycelial form [29], but a subsequent study 
confirmed the expression of this antigen in the yeast form as well [2]. Our results were in agreement with that observation; the presence of CR3-RP was confirmed in hyphae of C. albicans SC5314, but also in the yeast form of $C$. auris 0390. Thus, CR3-RP may represent an attractive target for prevention and treatment of infections associated with different Candida spp. including C. auris [18].

\section{Activity of anti CR3-RP Ab against $C$. auris biofilms}

Currently, fungal surface antigens are involved in the study of novel approaches for the treatment of candidiasis, including vaccine development [22-24, 30]. Taking into account this information, we were interested in examining the potential activity of anti-CR3-RP Ab against resistant $C$. auris biofilms. In this set of experiments, the effectiveness of anti-CR3-RP $\mathrm{Ab}$ on the $C$. auris biofilm compared to the activity of conventional antifungals was studied. Results summarized in Fig. 2 demonstrate that incubation of yeasts in the presence of anti-CR3-RP Ab resulted in higher inhibition of biofilm formation compared to all antifungal agents tested (FLU, CAS, and AMB) in both C. albicans (Fig. 2a) and C. auris isolates (Fig. 2b-d). For all tested isolates, treatment with antiCR3-RP Ab resulted in statistically significant reduction in the metabolic activity of biofilm cells with $73 \%$ for C. auris 0390 , $36 \%$ for C. auris 0383 , and $49 \%$ for C. auris 0386 , compared

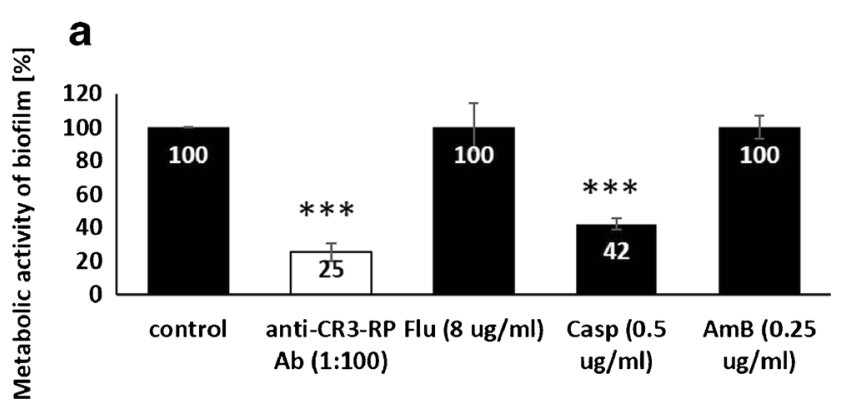

C. albicans SC5314

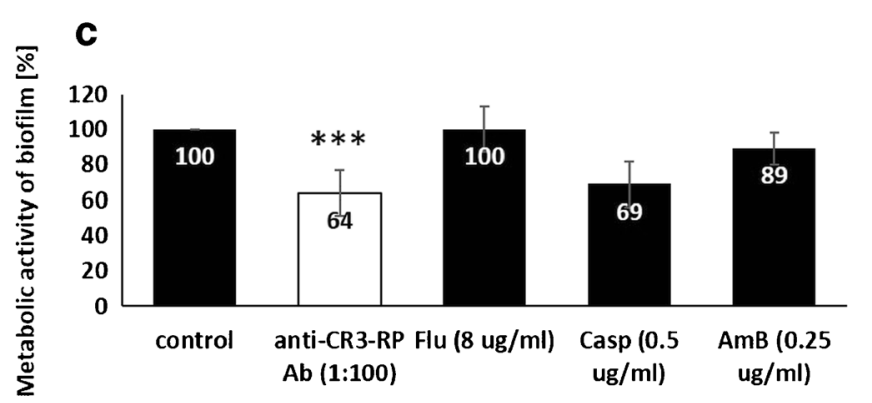

\section{C. auris 0383}

Fig. 2 Activity of anti-CR3-RP Ab to inhibit biofilm formation by C. albicans SC5314 (a) and C. auris strains (b C. auris 0390; c C. auris 0383; d C. auris 0386). The anti-CR3-RP Ab (white columns) or antifungal drugs (gray columns) were added at $t=0 \mathrm{~h}$ in the adherence to the untreated controls. Inhibition of biofilm formation of the reference strain C. albicans SC5314 was $75 \%$, also confirming results from our previous work [8]. These levels of biofilm inhibition compared favorable to those obtained with current antifungals. CAS was the only drug able to inhibit biofilm of the $C$. albicans strain and only one C. auris (0386) isolate by more than $50 \%(p<0.001)$.

For the second modality treatment, anti-CR3-RP Ab was added to the 24-h pre-matured biofilms of $C$. auris and C. albicans. As it is shown in Fig. 3, treatment with anti-CR3$\mathrm{RP} A \mathrm{~b}$ resulted in statistically significant differences $(p<0.001)$ in metabolic activity of biofilm cells in all tested $C$. auris isolates, as well as in the control strain C. albicans SC53141, although as expected, these decreases were lower than those observed for inhibition of biofilm formation, never reaching $>50 \%$ inhibition. However, the activity of anti-CR3-RP Ab was higher compared to all three conventional antifungals tested.

The anti-biofilm effect of anti-CR3-RP Ab was also supported by brightfield microscopy observations (Fig. 4). Results showed significant reduction in biofilm density and quantity for both tested strains of C. auris (0390) and C. albicans SC5314 (control) not only when anti-CR3-RP $\mathrm{Ab}$ was added at $t=0 \mathrm{~h}$ (Fig. $4 \mathrm{~b}, \mathrm{e}$ ), but also when anti CR3-RP Ab was administered after $24 \mathrm{~h}$ to pre-formed biofilm (Fig. 4c, f). These observations were compared to the control biofilms developed in the absence of treatment (Fig. 4a, d).
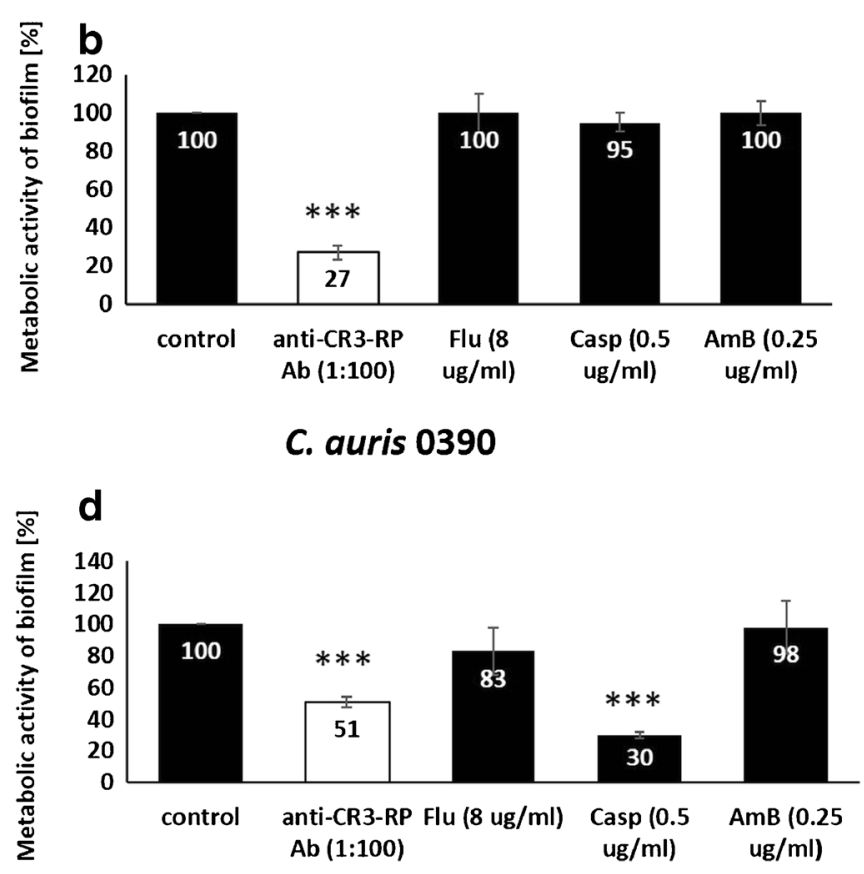

\section{C. auris 0386}

phase of biofilm formation. Cells within biofilms were significantly less metabolically active compared to the control after administration of $\mathrm{Ab}$ $(p<0.001(* * *))$ 


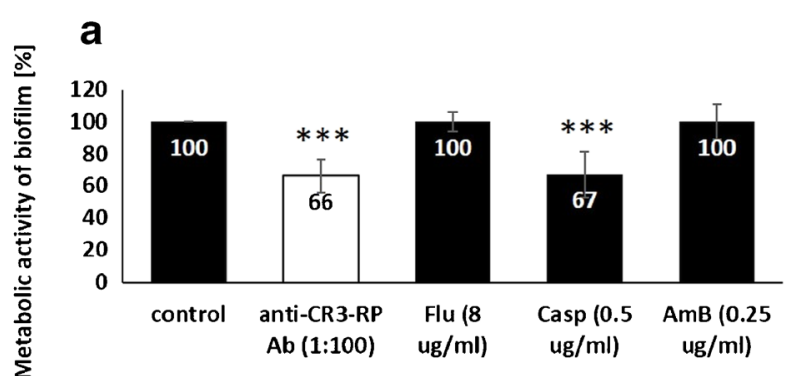

C. albicans SC5314

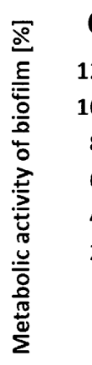

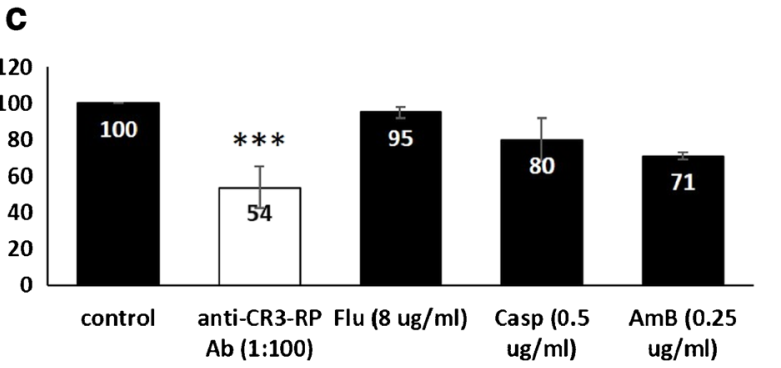

\section{C. auris 0383}

Fig. 3 Activity of anti-CR3-RP Ab against pre-formed mature biofilms of C. albicans SC5314 (a) and C. auris strains (b C. auris 0390; c C. auris 0383; d C. auris 0386). The anti-CR3-RP Ab (white columns) or antifungal drugs (gray columns) were added at $t=24 \mathrm{~h}$ after biofilms of

The strategy in which $\mathrm{Ab}_{\mathrm{s}}$ block target Candida surface proteins has been postulated in many studies as a promising alternative approach for combating Candida infection [22, 24, 30].

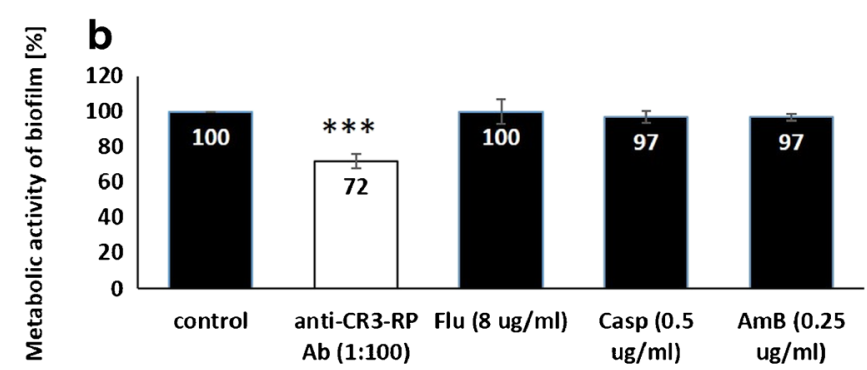

C. auris 0390

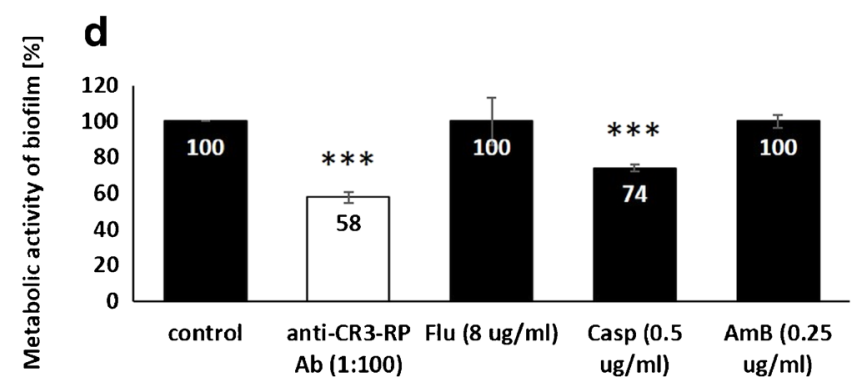

C. auris 0386

the different strains were fully formed. Cells within biofilms were significantly less metabolically active compared to the control after administration of $\mathrm{Ab}(p<0.001(* * *))$

Here, after demonstrating the presence of CR3-RP antigen in the C. auris cell wall, we also confirmed the effectiveness of antiCR3-RP Ab on multidrug-resistant biofilm formed by $C$. auris.

\section{C. albicans SC5314}
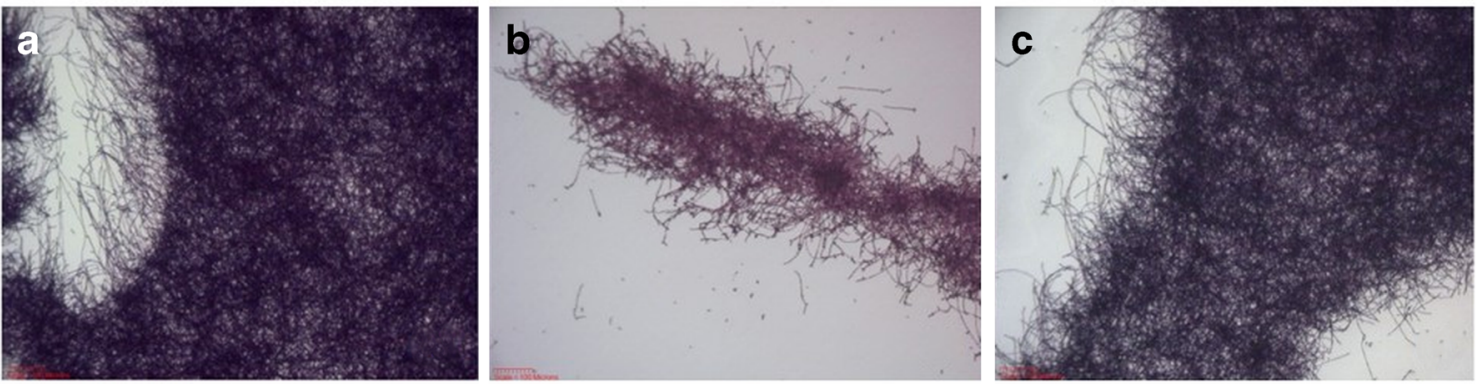

C. auris 0390
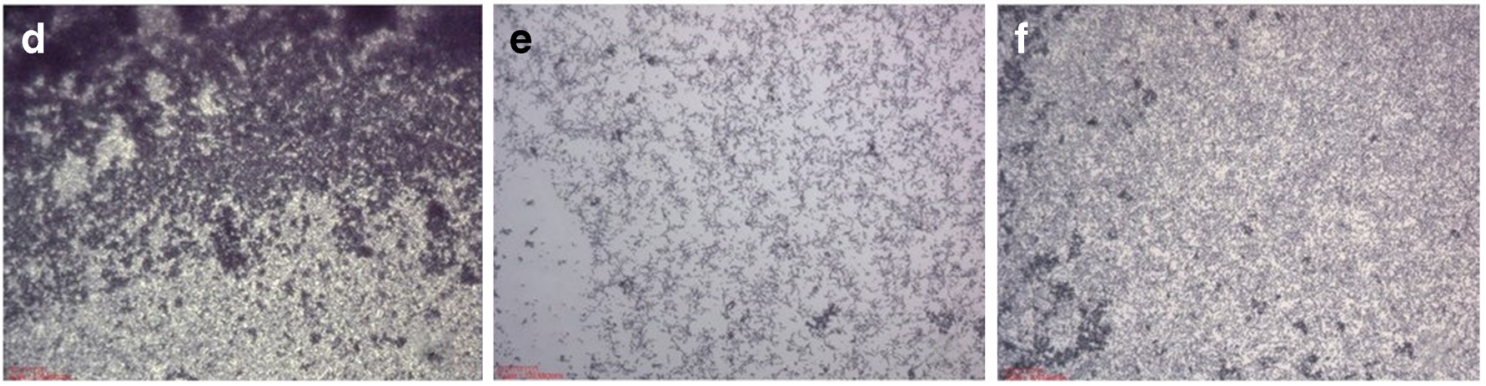

Fig. 4 Effect of anti-CR3-RP Ab on biofilms formed by C. albicans SC5314 (a-c) and C. auris $0390(\mathbf{d}-\mathbf{f})$ on polystyrene surface in vitro; control 48-h biofilm without antibody (a, d); biofilm formed in the presence of anti-CR3-RP Ab added in the adherence phase $(t=0 \mathrm{~h})(\mathbf{b}$, e); biofilm formed in the presence of anti-CR3-RP Ab added to preformed biofilm $(t=24 \mathrm{~h})(\mathbf{c}, \mathbf{f})$. Bars represent $100 \mu \mathrm{m}$. All samples were stained with $0.6 \%$ crystal violet 
The specific mechanism of action by which this $\mathrm{Ab}$ targets Candida spp., and in particular its activity against Candida biofilm, is unknown. It has been reported that anti-CR3-RP Ab can bind covalently to CR3-RP antigen in cell wall of Candida and through this mechanisms block the function of CR3-RP as a mimic protein, protecting the yeast against immune system reaction [7]. Possibility of cross reaction with other virulence proteins leading to decrease of adherence and biofilm formation could also be assumed. For example, Fujibayashi et al. (2009) described the blocking cell wall antigens involved in adhesion with 3 anti-Candida $\operatorname{IgY} \mathrm{Ab}_{\mathrm{s}}$ produced in egg yolk and suggested a possible cross-reaction of $\mathrm{Ab}_{\mathrm{s}}$ with Als3 and Hwp1 proteins leading to reduction in adhesion and biofilm development [31]. We could also hypothesize that binding of anti-CR3$\mathrm{RP} \mathrm{Ab}$ to its corresponding CR3-RP antigen in yeasts may lead to changes in the integrity of the cell wall or in cell surface properties, which could explain, at least in part, its activity against biofilms. However, more experiments are needed to confirm this hypothesis. Overall, our results demonstrated the activity of CR3-RP Ab against multidrug-resistant $C$. auris biofilms and may serve as the basis for the development of alternative strategy to tackle these difficult to treat infections caused by C. auris.

Acknowledgements The authors wish to thank Daniel MontelongoJauregui, MSc. (The University of Texas at San Antonio, TX, USA) for help with performing fluorescence microscopy of indirect immunofluorescence.

Funding This work was supported by the Slovak Research and Development Agency under contract no. [APVV-15-0347 to H. B.], and by the grant VEGA [1/0628/15 to H. B.] supported by the Ministry of Education, Science, Research and Sport of the Slovak Republic. This research was also supported by Fulbright Commission in Slovak Republic (J. D. was the Fulbright Visiting Student Researcher at J.L.L.R lab during period Sept 2017-Feb 2018). J.L.L.R acknowledges support by the Margaret Batts Tobin Foundation.

\section{Compliance with ethical standards}

Conflict of interest The authors declare that they have no conflict of interest.

Ethical approval Not applicable.

Informed consent Not applicable

\section{References}

1. Heidenreich F, Dierich MP (1985) Candida albicans and Candida stellatoidea, in contrast to other Candida species, bind iC3b and C3d but not C3b. Infect Immun 50:598-600

2. Bujdáková H, Würzner R, Klobušický M, Dierich MP (1997) Expression and quantification of the iC3b-binding protein in different Candida albicans strains and their morphological stages. FEMS Immunol Medical Microbiol 18:147-152
3. Paulovičová E, Bystrický S, Machová E, Bujdáková H (2008) Immune responsiveness of a novel peptidoglycan conjugate prepared from surface Candida immunogens: mannan and CR3related protein. FEMS Immunol Med Microbiol 53:421-428

4. Calderone RA (1995) Recognition of endothelial cells by Candida albicans: role of complement-binding proteins. Can J Bot 73:1154 1159

5. Hostetter MK (2008) The iC3b receptor of Candida albicans and its role in pathogenesis. Vaccine 26:108-1012

6. Bujdáková $\mathrm{H}$, Paulovičová E, Borecká-Melkusová S et al (2008) Antibody response to the $45 \mathrm{kDa}$ Candida albicans antigen in an animal model and potential role of the antigen in adherence. J Med Microbiol 57:1466-1472

7. Bujdáková H, Paulovičová E, Paulovičová L, Šimová Z (2010) Participation of the Candida albicans surface antigen in adhesion, the first phase of biofilm development. FEMS Immunol Med Microbiol 59:485-492

8. Chupáčová J, Borhi E, Morace G et al (2018) Anti-biofilm activity of antibody directed against surface antigen CR3-RP - comparison of Candida albicans and Candida dubliniensis. FEMS Pathog Dis 76(1). https://doi.org/10.1093/femspd/ftx127

9. Satoh K, Makimura K, Hasumi Y et al (2009) Candida auris sp. nov., a novel novel ascomycetous yeast isolated from the external ear canal of an inpatient in a Japanese hospital. Microbiol Immunol 53:41-44

10. Chowdhary A, Sharma C, Meis JF (2017) Candida auris: a rapidly emerging cause of hospital-acquired multidrug-resistant fungal infections globally. PLoS Pathog 13(5):e1006290 https://doi.org/10. 1371/journal.ppat.1006290

11. Dolande M, Garcia N, Capote AM et al (2017) Candida auris: antifungal multi-resistant emerging yeast. Curr Fungal Infect Rep 11:197-202

12. Larkin E, Hager C, Chandra J et al (2017) The emerging pathogen Candida auris: growth phenotype, virulence factors, activity of antifungals, and effect of SCY-078, a novel glucan synthesis inhibitor, on growth morphology and biofilm formation. Antimicrob Agents Chemother 61:e02396-e02316. https://doi.org/10.1128/ AAC.02396-16

13. Sherry L, Ramage G, Kean R et al (2017) Biofilm-forming capability of highly virulent, multidrug-resistant Candida auris. Emerg Infect Dis 23(2):328-331

14. Fakhim H, Vaezi A, Dannaoui E et al (2018) Comparative virulence of Candida auris with Candida haemulonii, Candida glabrata and Candida albicans in murine model. Mycoses 2018:1-6

15. Chatterjee S, Alampalli S, Nageshan RK et al (2015) Draft genome of commonly misdiagnosed multidrug resistant pathogen Candida auris. BMC Genomics 16:686

16. Sharma D, Kumar N, Pandey R et al (2016) Whole genome sequencing of emerging multidrug resistant Candida auris isolates in India demonstrates low genetic variation. New Microbes New Infect 13:77-82

17. Munoz JF, Gade L, Chow NA, et al (2018) Genomic basis of multidrug resistance mating and virulence in Candida auris and related emerging species. bioRxiv 299917. https://doi.org/10.1101/299917

18. Sears D, Schwartz BS (2017) Candida auris: an emerging multidrug-resistant pathogen. Int J Infect Dis 63:95-98

19. Coleman DA, Oh SH, Zhao X et al (2009) Monoclonal antibodies specific for Candida albicans ALS3 that immunolabel fungal cells in vitro and in vivo and block adhesion to host surfaces. J Microbiol Methods 78:71-78

20. Mishra NN, Ali S, Shukla PK (2015) A monoclonal antibody against $47.2 \mathrm{kDa}$ cell surface antigen prevents adherence and affects biofilm formation of Candida albicans. World J Microbiol Biotechnol 31:11-21

21. Torosantucci A, Tumbarello M, Bromuro C et al (2017) Antibodies against a $\beta$-glucan-protein complex of Candida albicans and its potential as indicator of protective immunity in candidemic 
patients. Sci Rep 7:2722. https://doi.org/10.1038/s41598-01702977-6

22. Gillum AM, Tsay EY, Kirsch DR (1984) Isolation of the Candida albicans gene for orotidine-5'-phosphate decarboxylase by complementation of S. cerevisiae ura3 and E. coli pyrF mutations. Mol Gen Genet 198:179-182

23. Ramage G, Van de Walle K, Wickes BL, Lopez-Ribot JL (2001a) Standardized method for in vitro antifungal susceptibility testing of Candida albicans. Antimicrob Agents Chemother 45(9):2475-2479

24. Bujdáková H, Lell C, Gruber A et al (1999) The influence of subinhibitory concentrations of conventional and experimental antifungal drugs on the expression of the $\mathrm{iC} 3 \mathrm{~b}$ binding protein in Candida albicans strains during filamentation. Pathog Dis 26(1):1-10

25. Lohse MB, Gulati M, Johnson AD, Nobile CJ (2018) Development and regulation of single and multi-species Candida albicans biofilms. Nat Rev Microbiol 16(1):19-31

26. Ramage G, Vandewalle K, Wickes NL, López-Ribot JL (2001b) Characteristics of biofilm formation by Candida albicans. Rev Iberoam Micol 18:163-170
27. Ramage G, Rajendran R, Sherry L, Williams C (2012) Fungal biofilm resistance. Int J Microbiol 2012:528521

28. Paulovičová E, Bujdáková H, Chupáčová J et al (2015) Humoral immune response to Candida albicans complement receptor 3related protein in the atopic subjects with vulvovaginal candidiasis. Novel sensitive marker for Candida infection. FEMS Yeast Res 15. https://doi.org/10.1093/femsyr/fou001

29. Alaei S, Larcher C, Ebenbichler C et al (1993) Isolation and biochemical characterization of the $\mathrm{iC} 3 \mathrm{~b}$ receptor of Candida albicans. Infect Immun 61:1395-1399

30. De Bernardis F, Amacker M, Arancia S et al (2012) A virosomal vaccine against candida vaginitis: immunogenicity, efficacy and safety profile in animal models. Vaccine 30(30):4490-4498

31. Fujibayashi T, Nakamura M, Tominaga A et al (2009) Effects of IgY against Candida albicans and Candida spp. adherence and biofilm formation. Jpn J Infect Dis 62:337-342 